TITLE:

\title{
Kinetic effects on the ideal pressure-driven modes in an $\mathrm{L}=2$ heliotron
}

\section{$\operatorname{AUTHOR}(\mathrm{S})$ :}

Yamagishi, O; Nakamura, Y; Kondo, K; Nakajima, N

\section{CITATION:}

Yamagishi, O ... [et al]. Kinetic effects on the ideal pressure-driven modes in an L=2 heliotron. PHYSICS OF PLASMAS 2003, 10(7): 2871-2880

\section{ISSUE DATE:}

2003-07

URL:

http://hdl.handle.net/2433/50251

\section{RIGHT:}

Copyright 2003 American Institute of Physics. This article may be downloaded for personal use only. Any other use requires prior permission of the author and the American Institute of Physics. 


\title{
Kinetic effects on the ideal pressure-driven modes in an $L=2$ heliotron
}

\author{
Osamu Yamagishi, ${ }^{a}$ Yuji Nakamura, and Katsumi Kondo \\ Graduate School of Energy Science, Kyoto University, Uji, Kyoto 611-0011, Japan \\ Noriyoshi Nakajima \\ National Institute for Fusion Science, Toki, Gifu 509-5292, Japan
}

(Received 10 February 2003; accepted 28 April 2003)

\begin{abstract}
The kinetic effects on the ideal ballooning and interchange/Mercier modes are studied in model equilibria for an $L=2$ heliotron, large helical device (LHD) [A. Iiyoshi et al., Nucl. Fusion 39, 1245 (1999)]. It is shown that the ion finite Larmor radius (FLR) effect stabilizes the modes with high toroidal mode number, $n$. On the other hand, the finite electron compressibility plays a double role, and stabilizes the low- $n$ modes as the ideal magnetohydrodynamic (MHD) modes, while it destabilizes the high- $n$ modes. It is discussed that the inclusion of the compressibility impacts the stability, and this effect is stronger in LHD than in a comparable tokamak, which is due to the larger magnitude of the local curvature. As a result of the competition between the FLR and the compressibility, it is shown in LHD that the low- $n$ instabilities can become much weaker than that expected by the ideal MHD, while the high- $n$ instabilities are prone to remain unstable near the plasma core region. (C) 2003 American Institute of Physics. [DOI: 10.1063/1.1584682]
\end{abstract}

\section{INTRODUCTION}

Pressure-driven instabilities are considered to suppress the increase of the plasma beta, so that the stability of such instabilities has been widely studied, both in tokamak and helical plasmas. Many of these are based on the system of ideal magnetohydrodynamic (MHD) equations. This is a single-fluid model, and consists of moment equations of the kinetic equation and the Maxwell equations. ${ }^{1}$ Since many assumptions are made to close the system of MHD equations, it is natural to start with a more exact formulation when the assumptions come into question and we want to investigate the more exact stability.

For this reason, one of the gyrokinetic, gyrofluid, or twofluid models is usually used to investigate the stability including kinetic effects. For example, a very exact formulation of the gyrokinetic model can be found in Ref. 2, in the framework of the linear local (ballooning) approximation. The mode equations are usually integro-differential equations owing to the velocity dependence in the kinetic model, and instead the assumptions for closure are made in the fluid model. Since it is rather complicated to solve these models numerically, mainstream analyses are concentrated on the electrostatic modes in the case of helical plasmas. ${ }^{3-8}$ The electrostatic modes are considered to belong to the drift branch, such as the ion temperature gradient mode (ITG), trapped electron mode (TEM), and so on. On the other hand, the pressure-driven modes that we are studying are known to belong to the shear-Alfvén branch, which are the electromagnetic modes.

The so-called kinetic ballooning mode (KBM) equation was derived by several authors, ${ }^{9-13}$ based on the electromagnetic kinetic/fluid models. In deriving the KBM equation,

${ }^{\text {a)} E l e c t r o n i c ~ m a i l: ~ y a m a g i s i @ n i f s . a c . j p ~}$ assumptions are imposed for the formal solution of the gyrokinetic equation for each species, to reduce the velocity integration in the formulation and to emphasis specific physics. Although there are many versions of KBM equations owing to the different assumptions, the resulting mode equation should include the counterpart of the MHD branch. In this spirit, a linear local KBM equation similar to the MHD ballooning mode equation was derived. ${ }^{9}$ It is beneficial to use the KBM equation when we want to see the kinetic effects on the ideal MHD modes, since the mode equation is such that terms related to the kinetic effects are added to the MHD mode equation. This is essentially a one-dimensional differential equation, so that the use of the KBM equation is also advantageous in the viewpoint of the numerical calculations to investigate the stability of the kinetic shear-Alfvén modes, as long as the assumptions used are valid. So far, many studies of the KBM stability have been done in the tokamak plasmas, ${ }^{10-20}$ while there are only a few studies in the helical plasmas. ${ }^{21}$

As is well known, the pressure-driven modes are classified into the ballooning and interchange/Mercier mode. It is often argued that the Mercier instability comes into question in the typical $L=2$ heliotron, because the outer radius region is prone to be in the magnetic hill. Thus the stability of the Mercier or interchange modes is regarded in the helical plasmas more than in the tokamak plasmas. Recently it was shown in the Large Helical Device (LHD) experiments that good particle confinement can be attained by shifting the magnetic axis inward $\left(R_{\mathrm{ax}}=3.6[\mathrm{~m}]\right) .{ }^{22}$ On the other hand, the inward shift of the axis makes the magnetic hill range broad. Thus the Mercier stability was investigated in detail theoretically. ${ }^{23}$ The observation of the low- $n$ MHD mode was also implemented, ${ }^{24}$ and the MHD activity was observed at typical low-order rational surfaces. However, although the experimental beta value has already been far over the theo- 

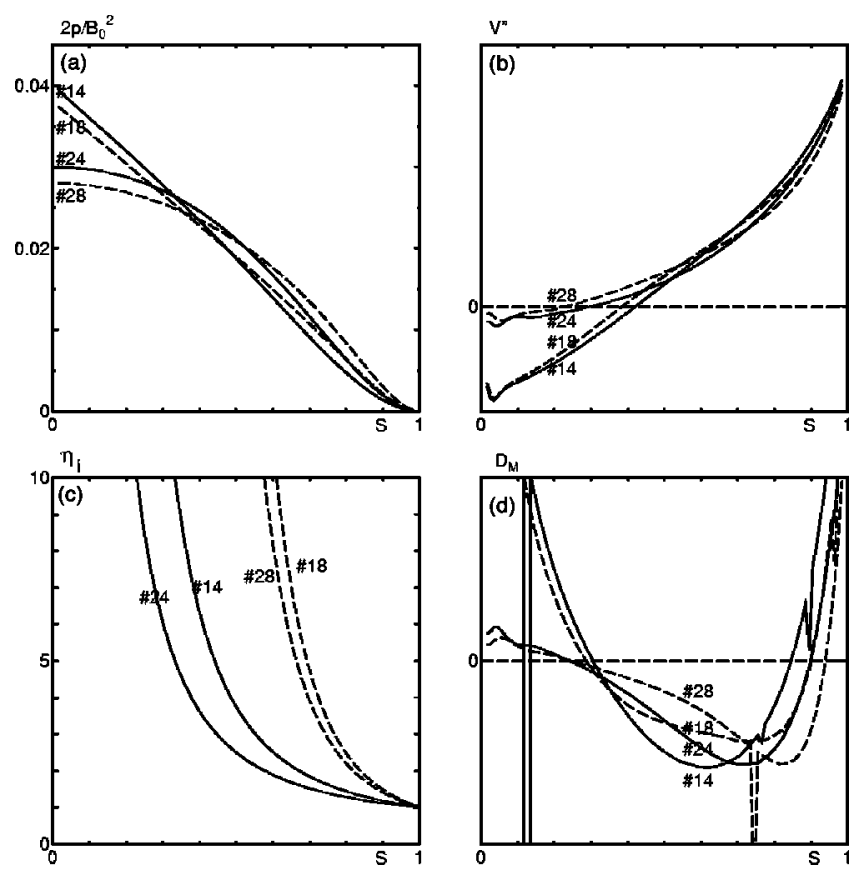

FIG. 1. Equilibrium quantities as a function of radial label $s$, in \#14, \#18, $\# 24$ and \#28 equilibrium at $\langle\beta\rangle \sim 1.8 \%$. (a) Pressure profile normalized by $B_{0}^{2} / 2 \mu_{0}$. (b) Measure of magnetic well ( $V^{\prime \prime}>0$ corresponds to the magnetic hill). (c) $\eta_{i}=d \ln T_{i} / d \ln n_{i}$. (d) Mercier coefficient $\left(D_{M}<0\right.$ indicates instability).

retical critical value for the Mercier mode, it has never been reported that the MHD activity collapses the plasma in the experiments. This suggests a potential that dynamics other than the ideal MHD are concerned with the activity in LHD. This is one of the motivations in this article, and the kinetic effects on the ideal MHD modes are studied, using an LHD configuration with $R_{\mathrm{ax}}=3.75[\mathrm{~m}]$ as a model equilibrium. ${ }^{25}$

In Sec. II, properties of LHD equilibria used in this study are shown, and the Mercier stability and the results of the quantization condition for the ideal modes are presented. In Secs. III-VII, the kinetic results are shown. The conclusions are given in Sec. VIII.

\section{MHD EQUILIBRIUM OF LHD}

In this section, we present the properties of the MHD equilibria of LHD used, and the stability of the ideal modes, in particular of the ideal interchange-like modes. The MHD equilibria with fixed boundary are calculated by the VMEC code. ${ }^{26}$ In the VMEC calculations, a pressure profile is needed as an input, which should be consistent with the density and temperature profile needed for the kinetic analysis in the next section. In this study, we simply assume $n_{i}=n_{e}$ $\equiv n_{0}$ and $T_{i}=T_{e} \equiv T$, so they are related to the pressure profile as $p=2 n_{0} T$. If, for example, $T / T(0)=1-s^{l}$ and $n_{0} / n_{0}(0)=1-s^{m}$ are assumed, then the pressure profile is given as $p / p(0)=1-s^{l}-s^{m}+s^{l+m}$. This pressure profile is used in the VMEC calculation assuming the net current is free. Here $s$ is the normalized toroidal flux function. We call \# $l m$ equilibrium for the above set of $(l, m)$.

In Fig. 1, the equilibrium quantities are shown for \#14, $\# 18$, \#24 and \#28 equilibrium, where the average beta is
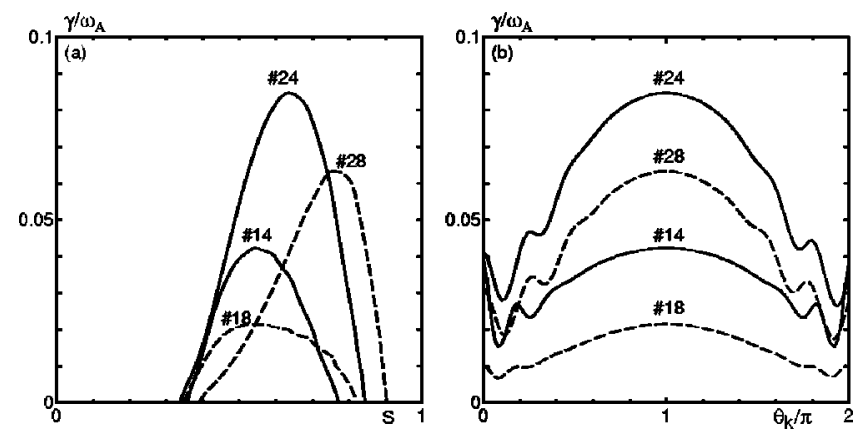

FIG. 2. Ideal growth rates in same equilibria as in Fig. 1. (a) Growth rate as a function of $s$ with $M \alpha=\pi$ and $\theta_{k}=\pi$ which correspond to interchange branch. (b) Growth rate as a function of $\theta_{k}$ with $M \alpha=\pi$. Radial label, $s$ is chosen so as to give maximum in right figure.

$\langle\beta\rangle \sim 1.8 \%$. We also assume $n_{0}(0)=3.0 \times 10^{+19}\left[\mathrm{~m}^{-3}\right]$ and $T(0)=2.0[\mathrm{keV}]$, values which seem to be routinely obtained in the experiments. ${ }^{27}$ Since the MHD equilibrium is labeled by the beta value and we specify the physical value of $2 n_{0}(0) T(0)$, the magnetic field strength is determined in the physical dimension. In the case of $\langle\beta\rangle \sim 1.8 \%, B_{0}$ $\sim 1.1[\mathrm{~T}]$ for $\# 14$ and \#18, and $B_{0} \sim 1.3[\mathrm{~T}]$ for \#24 and \#28 equilibrium. Here $R_{0}=3.9[\mathrm{~m}]$ is the major radius defined by the center of the helical coil and $B_{0}$ is toroidally averaged magnetic field at $R=R_{0}$. Since the density profile is assumed to be very flat in \#18 and \#28, $\eta_{i}=d \ln T / d \ln n_{0}$ becomes very large in the core region, as in Fig. 1(c). [When $n_{0} / T$ is constant $(l=m), \eta_{i}=1$ everywhere.] In all cases, there exists broad Mercier unstable range $\left(D_{M}<0\right)$ as in Fig. 1(d), which is due mainly to the magnetic hill, as in Fig. 1(b).

In Fig. 2, the ideal growth rate obtained by solving the ideal ballooning mode equation ${ }^{28}$ is plotted versus normalized toroidal flux, $s$ and ballooning parameter, $\theta_{k}$. Here the field line label $M \alpha$ is taken to be $\pi$, which corresponds to a field line passing through the utmost point from the major axis on the horizontally elongated poloidal cross section. Here $M$ is the number of the field periods in the toroidal direction, and $M=10$ for LHD. As shown in Fig. 2(a) where $\theta_{k}=\pi$ is assumed, the radial profile of growth rate is similar to the Mercier index $-D_{M}$, and this suggests that the local modes with $\theta_{k} \sim \pi$ correspond to the interchange (Mercier) branch in LHD. ${ }^{29-31}$ In fact, the correspondence of the Mercier unstable range and unstable range from ballooning equation with $\theta_{k}=\pi$ becomes better as we consider longer distance along the field line. ${ }^{32}$ This branch has weak $\alpha$ dependence so that the quantization condition $^{28}$ can be applied.

By using the quantization condition, the global toroidal mode number $n_{\text {tor }}$ is related to the quantized growth rate as ${ }^{28}$

$$
n_{\text {tor }}=2 \pi\left(N_{r}+\frac{1}{2}\right) / \Omega(\omega),
$$

where $N_{r}$ is a radial node number and is taken to be zero for the most unstable modes. Here $\Omega$ is determined by following a ray trajectory in the $\left(q, \theta_{k}, \alpha\right)$ space for a fixed $\omega$. The ray follows the ray equations ${ }^{28,29}$

$$
\dot{\alpha}=-\theta_{k} \frac{\partial \lambda}{\partial \theta_{k}}, \quad \dot{q}=\frac{\partial \lambda}{\partial \theta_{k}}, \quad \tilde{\theta_{k}}=\theta_{k} \frac{\partial \lambda}{\partial \alpha}-\frac{\partial \lambda}{\partial q},
$$



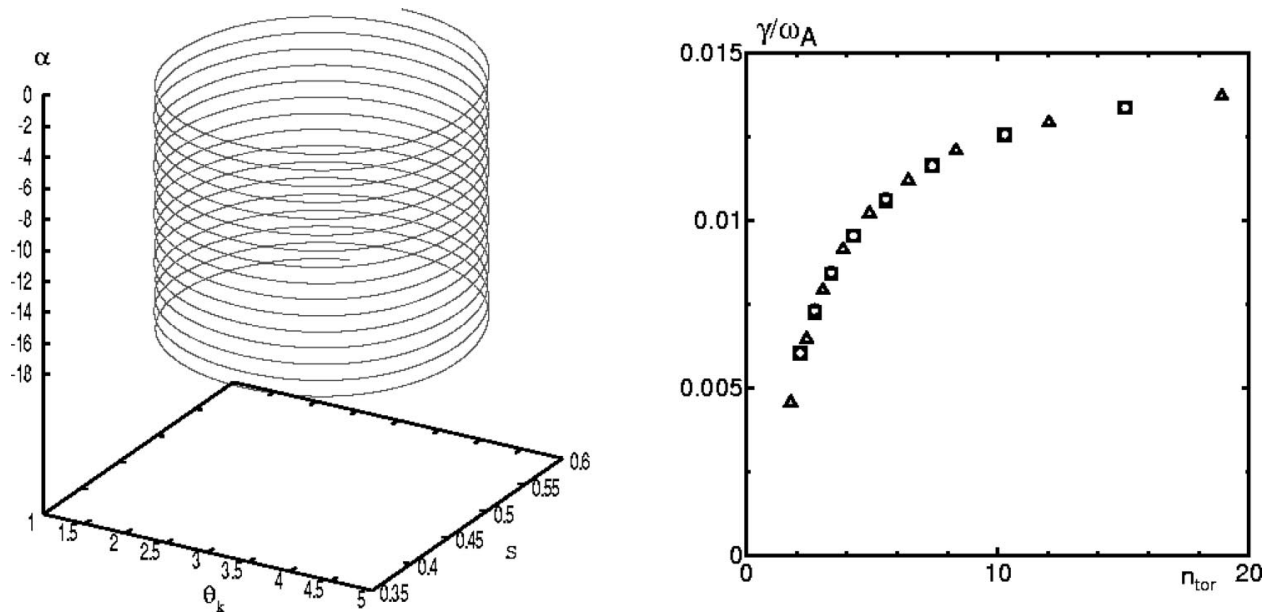

FIG. 3. (Left) Ray trajectory on $\gamma / \omega_{A}=0.0079$ isosurface. (Right) The ideal growth rate as a function of toroidal mode number, where $\Omega$ is obtained by 3D (squares), 2D (circles), and approximated 2D (triangles) formula. An LHD configuration with $p / p(0)=\left(1-s^{2}\right)^{2}$ pressure at $\langle\beta\rangle$ $\sim 1.0 \%$ is used. where $\lambda\left(q, \theta_{k}, \alpha\right)=\omega^{2}$ is the growth rate squared for the ideal modes, and the dot represents the derivative with respect to dummy time.

It is noted that the ballooning eigenvalue is obtained as $\left(\omega / \omega_{A}\right)^{2} \propto \rho_{m}(s) \omega^{2}$. Usually $\rho_{m}=m_{i} n_{0}=$ const is assumed when the quantization is applied in the MHD case. In our case with specifying density profile $n_{0}$, the quantization must be done for the growth rate normalized, not to the function of spatial variable, but to the constant, since the quantized growth rate is such as one defined for a whole plasma. We choose the normalizing factor as the Alfvén frequency at the axis, $\omega_{A}(0)$, so that the growth rate obtained is multiplied by $\sqrt{n_{0}(0) / n_{0}(s)}$.

In the axisymmetric case without $\alpha$ dependence, $\Omega$ is the area circled by the ray in the $q-\theta_{k}$ plane, $\Omega_{2 \mathrm{D}} \equiv \oint \theta_{k} d q$. In the $3 \mathrm{D}$ case, it is given by the average distance of the ray in the negative $\alpha$ direction, $\Delta \alpha$, while the ray circles once in the $q-\theta_{k}$ plane. It is clear from Eq. (2) that when $\alpha$ dependence of $\lambda$ is negligible, it reduces as $\Omega_{3 \mathrm{D}} \equiv \Delta \alpha \sim-\oint \dot{\alpha} d t$ $=\oint \theta_{k} \dot{q} d t=\oint \theta_{k} d q=\Omega_{2 \mathrm{D}}$. Thus if the $\alpha$ dependence of the eigenvalue is very weak, the $2 \mathrm{D}$ formula $\Omega_{2 \mathrm{D}}=\oint \theta_{k} d q$ will give a good estimate. Since $\Omega_{2 \mathrm{D}}$ is the area of ellipse in the $q$ - $\theta_{k}$ plane, we can estimate $\Omega$ more simply as follows. For a fixed $\alpha=\alpha_{0}$ and the center of interchange branch, $\theta_{k}=\theta_{k 0}$ $(=\pi)$. We calculate the local dispersion relation $\lambda\left(q, \theta_{k 0}, \alpha_{0}\right)=\omega^{2}$. This curve is usually parabolic on $q$, so that the interval $\Delta q$ between two zero points of function $y(q)=\lambda(q)-\lambda_{0}$ can be estimated for a fixed eigenvalue $\lambda_{0}$. Similarly for fixed $q=q_{0}$, such that the minimum (the most unstable) eigenvalue is given by $\operatorname{MIN}\left[\lambda\left(q, \theta_{k 0}, \alpha_{0}\right)\right]$ $=\lambda\left(q_{0}, \theta_{k 0}, \alpha_{0}\right)$, the local dispersion relation $\lambda\left(q_{0}, \theta_{k}, \alpha_{0}\right)$ $=\omega^{2}$ is calculated, and we obtain $\Delta \theta_{k}$. Then the area of ellipse for fixed $\lambda$ can be approximated by $\Omega \simeq \pi(\Delta q / 2)$ $\times\left(\Delta \theta_{k} / 2\right)$.

An example of the quantization result for the ideal mode is shown in Fig. 3, where an LHD equilibrium with $p / p(0)$ $=\left(1-s^{2}\right)^{2}$ at $\langle\beta\rangle=1.0 \%$ is considered. The squares and circles show the results using $\Omega_{3 \mathrm{D}}$ and $\Omega_{2 \mathrm{D}}$, respectively, and triangles show the approximated results with $\Omega$ $\simeq \pi(\Delta q / 2)\left(\Delta \theta_{k} / 2\right)$. In this equilibrium the $\alpha$ dependence of $\lambda$ is very weak, as shown by the ray trajectory in the left frame of Fig. 3, and it can be seen that the results using different methods to estimate $\Omega$ are reasonably on a curve. The last method is more practicable than the ray tracing, since it is still a hard task to obtain the local dispersion, $\lambda\left(s, \theta_{k}, \alpha\right)$ with fine meshes.

At this beta, $\langle\beta\rangle \sim 1.0 \%$, the ballooning branch near $\theta_{k}$ $\sim 0$ does not dominate yet, and the $\gamma$ is parabolic on $\theta_{k}$, as shown in Fig. 2(b). However, it can be confirmed that the ballooning branch arises with larger growth rate near $\theta_{k} \sim 0$ at higher beta. ${ }^{30,31}$ Thus when beta becomes so high that the ballooning branch breaks the parabolic $\gamma-\theta_{k}$ curve, the quantization may fail.

\section{KINETIC BALLOONING MODE EQUATION}

There are many versions of the kinetic ballooning mode (KBM) equations ${ }^{9-13,33}$ in the linear local approximation. The KBM equation is derived by substituting the formal solution of the gyrokinetic equation for the ion and electron, which is written in terms of the perturbed electromagnetic potentials, into the quasi-neutral condition and Ampère's law. In this study, we concentrate on modes in the intermediate frequency regime; $\omega_{t i}, \omega_{b i}<\omega<\omega_{t e}, \omega_{b e}$. Then $\omega_{t i} / \omega, \omega_{b i} / \omega, \omega / \omega_{t e}$ and $\omega / \omega_{b e}$ can be used as small expansion_parameters. Here $\omega_{t j} \sim v_{\mathrm{th}, j} / R_{0}$ and $\omega_{b j}$ $\sim \sqrt{\epsilon_{0}} v_{\text {th,j }} /\left(R_{0} / M\right)$ are the transit frequency and bounce frequency for helically trapped particles respectively, where $M$ is number of field periods, $\epsilon_{0}$ is the inverse aspect ratio, $v_{\mathrm{th}, j}=\sqrt{2 T_{j} / m_{j}}$ is the thermal velocity, $T_{j}$ is the temperature and $m_{j}$ is the mass, for species $j=e$ or $i$, electron or ion. In the following, $m_{i} / m_{e}=1836$ is assumed.

Then, the KBM equation valid in the intermediate regime is given as [Eq. (3.42) of Ref. 9]

$$
\begin{gathered}
\frac{d}{d \theta}\left[\mathcal{A} \frac{d \xi}{d \theta}\right]+\frac{1}{B^{\theta}}\left[2 \mu_{0} p^{\prime} \kappa_{d} \xi+\frac{\mathcal{A}}{B^{\theta}} I \xi-\frac{\mu_{0} p}{1+\tau}\left\{\left(\kappa_{d}\right)^{2} G \xi\right.\right. \\
\left.\left.+\tau \kappa_{d} H \int_{1 / B_{\max }}^{1 / B_{\min }} \frac{d \Lambda \Lambda B^{2}}{\sqrt{1-\Lambda B}}\left\langle\kappa_{d} \xi\right\rangle\right\}\right]=0,
\end{gathered}
$$

where

$$
I(\bar{\omega})=\bar{\omega}^{2} \alpha_{1 i},
$$




$$
\begin{aligned}
& G(\bar{\omega})=7 \alpha_{2 i}+4 \tau \alpha_{1 i} \alpha_{1 e} / \alpha_{0 e}, \\
& H(\bar{\omega})=\left(\frac{15}{8}\right) \alpha_{2 e}+\left(\frac{1}{2}\right)\left(\alpha_{1 i}-3 \alpha_{1 e}\right) \alpha_{1 e} / \alpha_{0 e}, \\
& \alpha_{l j}=1-\bar{\omega}_{* j}\left(1+l \eta_{j}\right) / \bar{\omega}, \\
& \mathcal{A}=\left(\chi^{\prime 2} B^{\theta} / B^{2}\right)\left|\mathbf{k}_{\perp} / k_{\alpha}\right|^{2}, \\
& \mathbf{k}_{\perp} / k_{\alpha}=\nabla \zeta-q \nabla \theta-q^{\prime}\left(\theta-\theta_{k}\right) \nabla s, \\
& \kappa_{d}=-\frac{\chi^{\prime}}{B^{2}} \mathbf{B} \times \boldsymbol{\kappa} \cdot \mathbf{k}_{\perp} / k_{\alpha}=\kappa_{n}+\kappa_{g} q^{\prime}\left(\theta-\theta_{k}\right), \\
& \omega_{* j}=\frac{T_{j}}{B^{2} e_{j} n_{j}} \mathbf{B} \times \nabla n_{j} \cdot \mathbf{k}_{\perp}=-n_{k} \frac{T_{j}}{e_{j} \chi^{\prime}} \frac{n_{j}^{\prime}}{n_{j}} .
\end{aligned}
$$

Here $\mu_{0}$ is the permeability in vacuum, $s$ is the normalized toroidal flux, $s=\psi / \psi_{\text {edge }}, 2 \pi \chi$ is the poloidal flux, and $\theta, \zeta$ are usual Boozer angle coordinates. ${ }^{34} q=\psi^{\prime} / \chi^{\prime}$ is the safety factor, $B$ is the magnetic field strength, $B^{\theta}=\mathbf{B} \cdot \nabla \theta$ $=\chi^{\prime} / \sqrt{g}, \kappa_{n}=\boldsymbol{\kappa} \cdot \sqrt{g}(\nabla \theta \times \nabla \zeta), \boldsymbol{\kappa}_{g}=\boldsymbol{\kappa} \cdot \sqrt{g}(\nabla s \times \nabla \theta)$ with curvature vector $\boldsymbol{\kappa}=(\mathbf{B} / B) \cdot \nabla(\mathbf{B} / B)$, and $\sqrt{g}=(\nabla s \times \nabla \theta$ $\cdot \nabla \zeta)^{-1}$ is the Jacobian to the Boozer coordinates. Prime denotes the derivative w.r.t $s$, and $d / d \theta=\partial / \partial \theta+q \partial / \partial \zeta$ is the derivative along a field line. The toroidal mode number $n_{k}$ $=k_{\alpha}$, which should be given as an input, enters through the diamagnetic frequency, $\omega_{* j}$. The density for species $j$ is denoted by $n_{j}$, and $\tau=T_{e} / T_{i}, \eta_{j}=d \ln T_{j} / d \ln n_{j}, \Lambda=\mu / E$ is the pitch angle variable with $\mu=v_{\perp}^{2} /(2 B)$ and $E=v^{2} / 2$ being the magnetic moment and the kinetic energy of a particle (divided by $\left.m_{j}\right),\langle\cdot\rangle$ denotes bounce average, and $\langle\cdot\rangle \equiv \int_{\theta_{1}}^{\theta_{2}} d \theta\left[\cdot B /\left(B^{\theta} v_{\|}\right)\right] / \int_{\theta_{1}}^{\theta_{2}} d \theta\left[B /\left(B^{\theta} v_{\|}\right)\right] \quad$ with $v_{\|}$ $=\sqrt{2 E(1-\Lambda B)}$, where all the sets of turning points along the field line $\left(\theta_{1}, \theta_{2}\right)$ are taken into account for a fixed $\Lambda$. The overbar denotes the normalization for frequencies to the Alfvén frequency; $\bar{\omega}=\omega / \omega_{A}$ with $\omega_{A}=B_{0} /\left(\sqrt{\mu_{0} m_{i} n_{i}} R_{0}\right)$, and the magnetic field, the length, the current and the flux are normalized to $B_{0}, R_{0}, R_{0} B_{0}$ and $R_{0}^{2} B_{0}$, respectively. Here $R_{0}$ is the major radius defined by the center of the helical coil and $B_{0}$ is toroidally averaged magnetic field at $R=R_{0}$. The second term is a pressure-driven term, which is the same as that of the ideal model, and the third term represents the inertia term. The fourth term is related to the fluid compressibility, ${ }^{12}$ and the fifth term represents the effect of the trapped electrons. In order to obtain Eq. (3), $\omega_{d i} / \omega$, $\left(k_{\perp} \rho_{i}\right)^{2} / 2$ and $\beta$ are also assumed to be smaller than unity so that their effects (ion magnetic drift and ion Larmor radius) can be treated as small perturbations. Here $\omega_{d j}$ is given in the Boozer coordinates as $\omega_{d j}=-n_{k}\left[E /\left(\chi^{\prime} \Omega_{j} B\right)\right]\left[B^{2} \kappa_{d}(2\right.$ $\left.-\Lambda B)-\mu_{0} p^{\prime} \Lambda B\right], \quad$ and $\quad \rho_{i} \sim \rho_{\mathrm{th}, i}=v_{\mathrm{th}, i} / \Omega_{i} \quad$ with $\Omega_{j}$ $\equiv e_{j} B / m_{j}$ being the Larmor frequency of species $j$. This is valid for the MHD-like kinetic modes whose mode frequency is of the order of $\omega_{A} \cdot{ }^{9}$

If we ignore the trapped electron term, Eq. (3) can be written in the form

$$
d \mathcal{X} / d \theta=-\mathcal{K} \xi, \quad d \xi / d \theta=\mathcal{X} / \mathcal{A},
$$

which is integrated by the fourth-order Runge-Kutta method, from the boundaries $\theta= \pm \theta_{\max }$ with the boundary condition $\xi\left( \pm \theta_{\max }\right)=0$ and small $d \xi\left( \pm \theta_{\max }\right) / d \theta$ to the mid
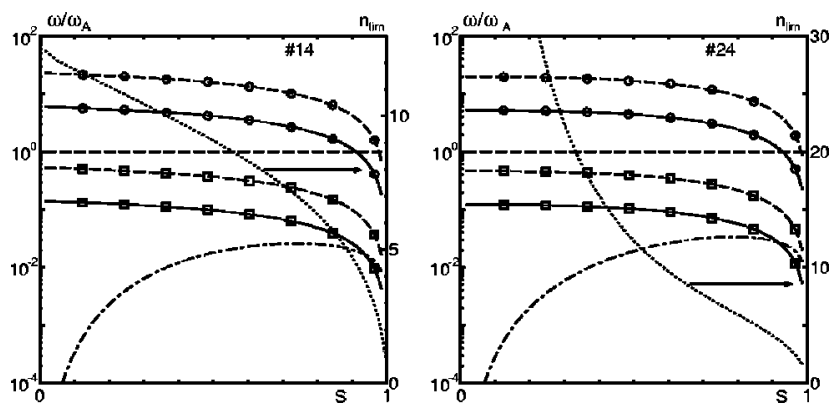

FIG. 4. Frequencies normalized to the Alfvén frequency in \#14 (left) and \#24 (right) equilibrium at $\langle\beta\rangle \sim 1.8 \%$. Transit and bounce frequencies are plotted by solid and dashed lines, respectively, for electron (ion) denoted by circles (squares), and ion diamagnetic frequency $\left|\omega_{* i}\right|$ with $n_{k}=1$ is shown by dash-dotted line. Critical $n_{\lim }$, over which the intermediate frequency regime can be satisfied, is also shown by dotted line for right axis of ordinate.

point $\theta=0$. The solutions $\xi$ and $\omega$ can be obtained by seeking the zeroth of $Z$ in the complex plane, defined by

$$
\begin{aligned}
Z(\omega) & \equiv\left[\frac{d \ln (\operatorname{Re} \xi)}{d \theta}\right]_{\theta=-0}^{\theta=+0}+i\left[\frac{d \ln (\operatorname{Im} \xi)}{d \theta}\right]_{\theta=-0}^{\theta=+0} \\
& =Z_{R}+i Z_{I},
\end{aligned}
$$

as changing $\omega=\omega_{r}+i \gamma$ as a complex parameter, which is done by a routine based on the Muller method. ${ }^{35}$ To accelerate the convergence, $\left(n_{\mathrm{z}}+1\right)^{a}$ is multiplied by $Z_{R}$ and $Z_{I}$ in each iteration of the $\omega$ scan, where $n_{\mathrm{z}}$ is the number of zeroth of the real or imaginary part of $\xi$ and $a$ is a real number near the unity, since we are interested in MHD-like modes with zero node along a field line. By this constraint, more weak modes such as what they call $\eta_{i}$-driven $\mathrm{KBM}^{13}$ may be eliminated from the analysis because they tend to be more broad and oscillate along a field line.

In order to check the validity of frequency regime we assume, the associated frequencies are shown in Fig. 4 for the \#14 and \#24 equilibrium in the previous section. Here the transit and helically bounce frequencies are shown, in addition to the ion diamagnetic frequency with $n_{k}=1$. The condition, $\omega<\omega_{t e}, \omega_{b e}$ is usually fulfilled. On the other hand, $\omega_{t i}, \omega_{b i}<\omega$ maybe dubious when the mode frequency becomes low. As has often been discussed, ${ }^{9}$ the frequency becomes $|\omega| \gtrsim\left|\omega_{* p i} / 2\right|$ at the marginal stability. Thus we also plot $n_{\text {lim }}$ given by $n_{\text {lim }}=\omega_{t i} /\left(\left|\omega_{* p i} /\left(2 n_{k}\right)\right|\right)$, i.e., when $n_{k}$ $\gtrsim n_{\text {lim }}$, the intermediate regime, $\omega \gtrsim \omega_{t i}$, can be satisfied. In both equilibria, $n_{\lim } \sim 10$ at $s \sim 0.5$ so that it may be considered that the use of the equation is invalid in the low- $n$ regime. However, as shown later, the magnitude of the real frequency does not coincide with $\left|\omega_{* p i} / 2\right|$ exactly but becomes higher; $|\omega| \gtrsim\left|\omega_{* p i}\right|$. Thus $n_{\lim } \gtrsim 5$ would be required for the use of Eq. (3), except for the near core region. It is noted that the bounce frequency for helically trapped ions is rather high. This is because the $M$ number of LHD is large, $M=10$. Thus in some cases, it may be considered that the effects of trapped ions are significant. However, we will see that the effects of trapped particles are slight even for the trapped electrons. 
In the following, we solve the KBM equation numerically, including the terms up to the third or fourth in the left-hand side (LHS) of Eq. (3). The fifth term will be treated perturbatively.

\section{SIMPLIFID FLR CORRECTION TO THE IDEAL MODES}

The simplest kinetic model can be objectified by neglecting the last two terms in Eq. (3), where only the ion finite Larmor radius (FLR) effect is included. In this case, the only difference from the ideal ballooning equation assuming the incompressibility is the inertia term, i.e., $\omega^{2}$ in the ideal model is replaced by $\omega\left(\omega-\omega_{* p i}\right)$ in the kinetic model, with $\omega_{* p i} \equiv \omega_{* i}\left(1+\eta_{i}\right)$. We call this model MI-1 model, as in Ref. 10. Since the form of the equation is unchanged from the ideal one, $\omega\left(\omega-\omega_{* p i}\right)$ is purely real. From this, it can be understood that when the mode is unstable, $\omega_{r}=\omega_{* p i} / 2$ and $\gamma=\sqrt{\left(\gamma_{\mathrm{MHD}}\right)^{2}-\left(\omega_{* p i} / 2\right)^{2}}$ hold exactly. Here $\omega=\omega_{r}+i \gamma$, with $\omega_{r}$ being a real frequency and $\gamma$ being a growth rate, and $\gamma_{\mathrm{MHD}}$ is a growth rate for the corresponding ideal mode. In this model, the mode frequencies can be determined by $\omega_{* p i}$ and $\gamma_{\mathrm{MHD}}$ only, so that we do not necessarily calculate the frequencies in the complex plane. It is noted that the growth rate of the kinetic is mode never beyond that of the ideal mode in this model. In addition, the increase of $\left|\omega_{* i}\right|$ $\propto n_{k}$ and $\left|1+\eta_{i}\right|$ are simply stabilizing.

A difference between the kinetic and ideal MHD formulations is that the toroidal mode number $n_{k}$ must be specified for the former case. That is included in the diamagnetic frequency, which has $n_{k}$ and $s$ dependence, as shown in Eq. (3) and Fig. 4. Thus in order to obtain the quantization results, this number should be consistent with the quantized toroidal mode number $n_{\text {tor }}$. For example, for a fixed $n_{k}$, we can quantize the growth rate and obtain the $\gamma-n_{\text {tor }}$ curve, as in the ideal case shown in the previous section. On that curve, the only $\gamma\left(n_{\text {tor }}=n_{k}\right)$ point should be picked up as a quantized result. Changing $n_{k}$ and iterating this procedure, we obtain the finite $n$ dependence of the kinetic growth rate.

In Fig. 5, the quantized growth rate is shown for the MI-1 modes, as well as that for the ideal interchange modes, in the same equilibria as in Fig. 2. Here only interchange-like modes with weak $\alpha$ dependence are unstable, so that the quantization is applicable. For both the ideal and MI-1 modes, the quantized growth rates show the decreasing dependence with decreasing $n_{\text {tor }}$, which is a typical result of quantization. In addition, as is usual for the FLR stabilization, the stabilizing FLR effect reduces the growth rate of the MI- 1 modes as $n_{\text {tor }}$ becomes high, As a result, it is shown that the $\gamma-n_{\text {tor }}$ curve for the kinetic mode has a peak, whereas the ideal mode becomes more unstable with $n_{\text {tor }}$. It is noted that for the equilibrium with parabolic $T$ profile (\#14 and \#18), $T \propto 1-\rho^{2}=1-s$, the quantized MI-1 modes are almost stabilized. This is because there are no intersecting points of $\gamma-n_{\text {tor }}$ curve and $n_{\text {tor }}=n_{k}=$ const. This is a result of the kinetic stabilization, and the difference between the configurations is due mainly to the different ideal growth rates, as shown in Fig. 2.

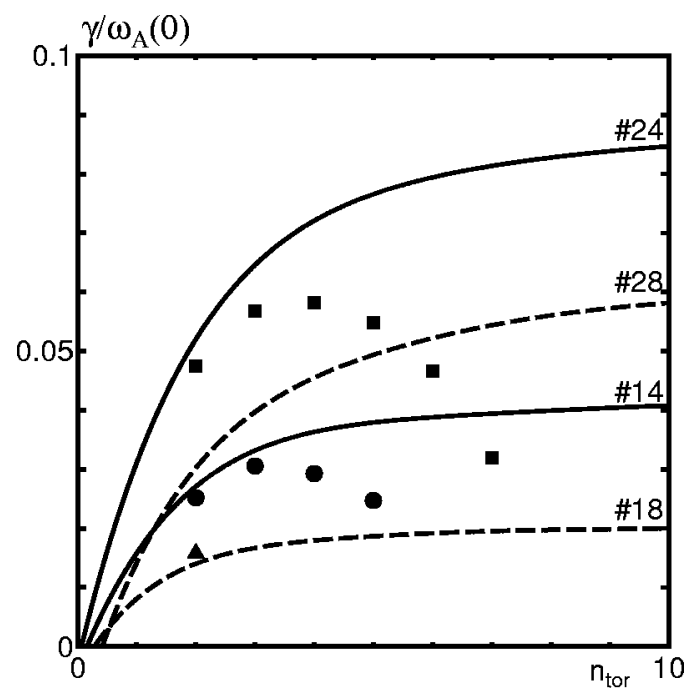

FIG. 5. Quantized growth rate as a function of finite toroidal mode number. Solid and dashed lines represent the growth rate for the ideal MHD (interchange) modes, and squares, circles, and triangles are shown for the MI-1 modes at $\langle\beta\rangle \sim 1.8 \%$ in $\# 24$, \#28, and \#14 equilibria, respectively. (The MI-1 modes in \#18 are stabilized.)

The MI-1 model is very simple, but only the stabilizing effect is included. More exact results including the fourth term in Eq. (3) are shown in the next section.

\section{COMPRESSIBLE EFFECT ON THE KINETIC MODES}

Here we consider the cases including the fourth term in Eq. (3). It has been discussed in Refs. 12 and 36 that this term is related to the perpendicular fluid compression in the fluid model. In fact, the fourth term represents the finite fluid compression approximately by ignoring the parallel compression in the ideal MHD energy integral: ${ }^{1} \gamma_{\mathrm{h}} p|\nabla \cdot \xi|^{2}$ $\simeq \gamma_{\mathrm{h}} p\left|\nabla \cdot \boldsymbol{\xi}_{\perp}\right|^{2} \neq 0$, together with a plausible assumption of vanishing magnetic compression, $\left(B^{2} / \mu_{0}\right)\left|\nabla \cdot \boldsymbol{\xi}_{\perp}+2 \boldsymbol{\kappa} \cdot \boldsymbol{\xi}_{\perp}\right|^{2}$ $\simeq 0$. Then in the MHD limit $n_{k}=0$, the fourth term is identified with the perpendicular compressibility, for a realistic specific heat ratio, $\gamma_{\mathrm{h}}=(7+4 \tau) /(4+4 \tau) .{ }^{9}$ We call this model the MI model, as in Ref. 10.

In this section, the \#24 equilibrium is used, which has broader pressure profile than that observed in typical experiments so that a strong instability is expected. In Fig. 6, the $n_{k}$ dependence of frequencies is shown for the ballooning branch with $\theta_{k}=0$ (squares) and the interchange branch with $\theta_{k}=\pi$ (circles), at $\langle\beta\rangle \sim 3.6 \%$ and $s=0.645$. The solid (dashed) line corresponds to MI (MI-1) model. The corresponding ideal growth rate is also shown by dash-dotted line, which formally corresponds to the $n_{k}=0$ in the MI- 1 model. As is noted in the previous section, the MI-1 mode is more stable than the ideal mode for all $n_{k}$, and the growth rate of the MI-1 mode monotonically decreases with $n_{k}$. On the other hand, it can be seen that $\gamma-n_{k}$ curves of the growth rate for the MI mode have a peak, and the growth rates can exceed that of ideal modes for specific $n_{k}$ spectrum range.

As can be seen from the comparison of MI-1 and MI growth rates, the inclusion of the compressible term can be- 

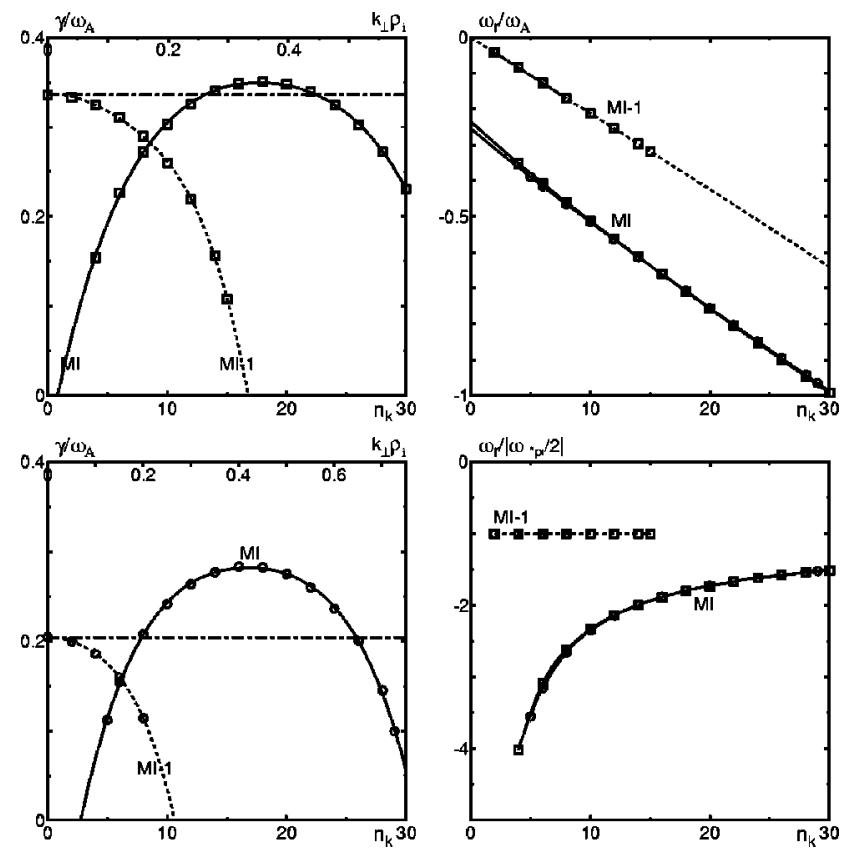

FIG. 6. Frequencies as a function of $n_{k}$ in LHD with \#24 pressure profile at $\langle\beta\rangle \sim 3.6 \%$, at $s=0.645$ and $M \alpha=\pi$. The frequencies for the MI- 1 modes are plotted by dashed lines, and that of the MI modes are plotted by solid lines. The dash-dotted lines show the ideal growth rate. Ballooning branch $\left(\theta_{k}=0\right)$ is marked by squares, and interchange branch $\left(\theta_{k}=\pi\right)$ is marked by circles. $k_{\perp} \rho_{i}\left(\theta=\theta_{k}\right)$ is also shown as the upper transverse axis in the figures for the growth rates.

come both stabilizing and destabilizing to the MI-1 modes, depending on the range of $n_{k}$. For the low $n_{k}$, it is stabilizing against the MI-1 mode, and it turns into destabilizing when the $n_{k}$ becomes high. Numerically, this can be understood from the function $G$ in Eq. (3), as noticed in Ref. 21 and originally emphasised in Ref. 9. For $n_{k}=0$, which formally corresponds to the ideal modes, $G$ becomes $7+4 \tau$, which is positive and so stabilizing. Therefore for low $n_{k}$, the inclusion of the compressibility is stabilizing, as is usual for the ideal modes. Oppositely, for the modes near the marginal stability with higher $n_{k}$, with $\gamma \sim 0$ and $\omega_{r} \sim \omega_{* p i} / 2$, the function $G$ becomes $G \sim-\left[7\left(1+3 \eta_{i}\right) /\left(1+\eta_{i}\right)+4 \tau(1\right.$ $\left.\left.+\eta_{i}\right)(1+2 \tau) /\left(1+\eta_{i}+2 \tau\right)\right]$, which is negative and so destabilizing. Therefore it follows that the critical $n_{k}$ over which the MI modes are stabilized is always higher than that of MI-1 modes.

It should be noted that the MI growth rates of the interchange-like modes can exceed that of the ideal modes sufficiently, while that of the ballooning-like modes is at most comparable to that of ideal one, at the peak of $\gamma-n_{k}$ curve. The real frequencies are found to be not so different for both the interchange and ballooning branches. Thus it is considered that the real frequency is not sensitive to the mode structure, and it is almost determined by $\omega_{* p i}$ and equilibrium geometry. It is shown that the real frequencies of the MI mode approach that of MI-1 mode, $\omega_{r} \sim \omega_{* p i} / 2$, as the $n_{k}$ increases. That is, the real frequency of the MI modes becomes dispersionless toward the marginal stability, as does that of the MI-1 modes.

In Fig. 7, the $\theta_{k}$ dependence of the MI frequencies with
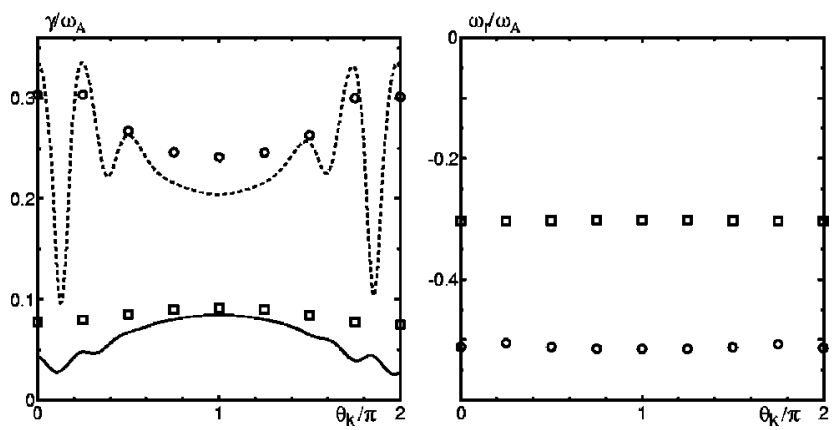

FIG. 7. Frequencies as a function of $\theta_{k}$ in \#24 equilibrium at $s=0.645$ with $M \alpha=\pi$. The MI frequencies with $n_{k}=10$ are shown by circles for $\langle\beta\rangle$ $\sim 3.6 \%$ and squares for $\langle\beta\rangle \sim 1.8 \%$. The ideal growth rate is also shown by a dashed line $(\langle\beta\rangle \sim 3.6 \%)$ and a solid line $(\langle\beta\rangle \sim 1.8 \%)$.

$n_{k}=10$ at $\langle\beta\rangle \sim 1.8 \%$ (squares) and $\langle\beta\rangle \sim 3.6 \%$ (circles) are plotted. The growth rate of the ideal mode is also shown. At $\langle\beta\rangle \sim 1.8 \%$, the ballooning branch near $\theta_{k} \sim 0$ is still weak, so that the ideal modes are interchange-like independent of $\theta_{k}$. In this case, the MI modes can become more unstable than the ideal modes. At higher beta, $\langle\beta\rangle \sim 3.6 \%$, the ballooning branch near $\theta_{k} \sim 0$ is more unstable than the interchange branch for the ideal mode. In this case, the MI growth rate is more stable than the ideal growth rate, and this is true for almost all $n_{k}$, as seen in Fig. 6. As $\theta_{k}$ approaches $\pi$, the modes are more unstable than the ideal one, as the mode structure changes to be interchange-like. As a result, the $\theta_{k}$ dependence of the growth rate becomes weaker by including the kinetic compressible effects.

In Fig. 8, the $\alpha$ dependence of the MI frequencies with $n_{k}=10$ at $\langle\beta\rangle \sim 3.6 \%$ is shown for ballooning and interchange-like modes, together with the corresponding ideal growth rates. As is expected from the case of the ideal modes, the $\alpha$ dependence is stronger for the ballooning branch than for the interchange one. However, again, the dependence on the local parameter becomes weaker than that of ideal modes, for both ballooning and interchange branch.

In Fig. 9, the ideal and real parts of the MI eigenfunction are compared, for the ballooning $\left(\theta_{k}=0\right)$ and interchange $\left(\theta_{k}=\pi\right)$ branches. It is found that the eigenfunctions for the ideal and MI modes are similar, and the MI eigenfunctions
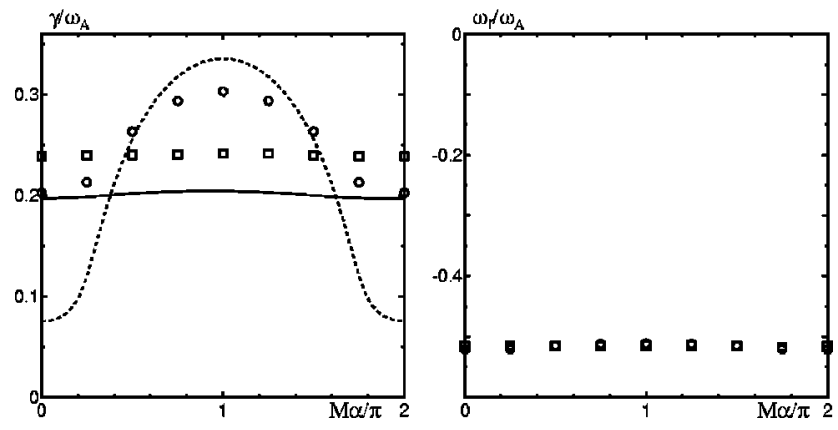

FIG. 8. Frequencies as a function of $\alpha$ in $\# 24$ equilibrium at $s=0.645$ at $\langle\beta\rangle \sim 3.6 \%$. The MI frequencies with $n_{k}=10$ are shown by circles for ballooning branch $\left(\theta_{k}=0\right)$ and squares for interchange branch $\left(\theta_{k}=\pi\right)$. The ideal growth rate is shown by a dashed line $\left(\theta_{k}=0\right)$ and solid line $\left(\theta_{k}\right.$ $=\pi)$. 


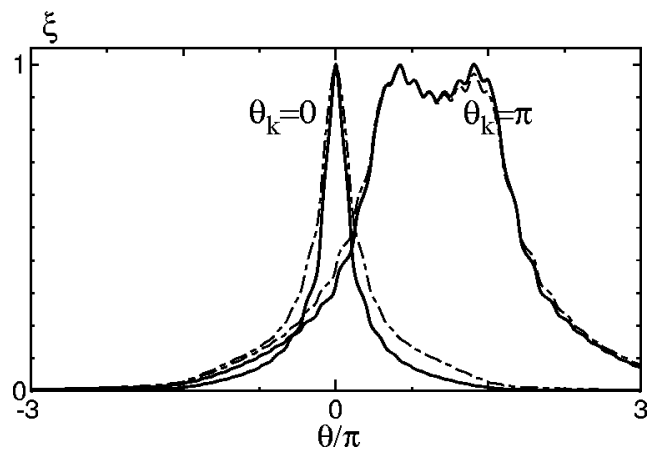

FIG. 9. Eigenfunction of ideal and MI modes belonging to ballooning branch $\left(\theta_{k}=0\right)$ and interchange branch $\left(\theta_{k}=\pi\right)$. The ideal mode is shown by a solid line, and real part of the MI mode with $n_{k}=10$ is shown by dash-dotted line. The equilibrium and the parameters are same as those in Fig. 6.

for both branches are somewhat broader than the ideal eigenfunctions by the compressible effects. This supports the above results because the dependence on the local parameters should be weak for more extended modes along a field line. In addition, since the ballooning and interchange mode is characterized by its localized and extended structure, the variation of growth rates from the ideal growth rates seems to be reasonable.

As can be seen in Fig. 6, the MI modes are stabilized with increasing $n_{k}$, at a fixed radial surface. Thus it is often concluded that the FLR effects can stabilize the modes easily. However, when we see the radial dependence of growth rates, the results are somewhat unexpected. In Fig. 10, the radial dependence of $\mathrm{MI}$ frequencies in the interchange branch are shown, in $\# 24$ equilibrium at $\langle\beta\rangle \sim 1.8 \%$, with different $n_{k}$. In this figure, the validity of the frequency re-
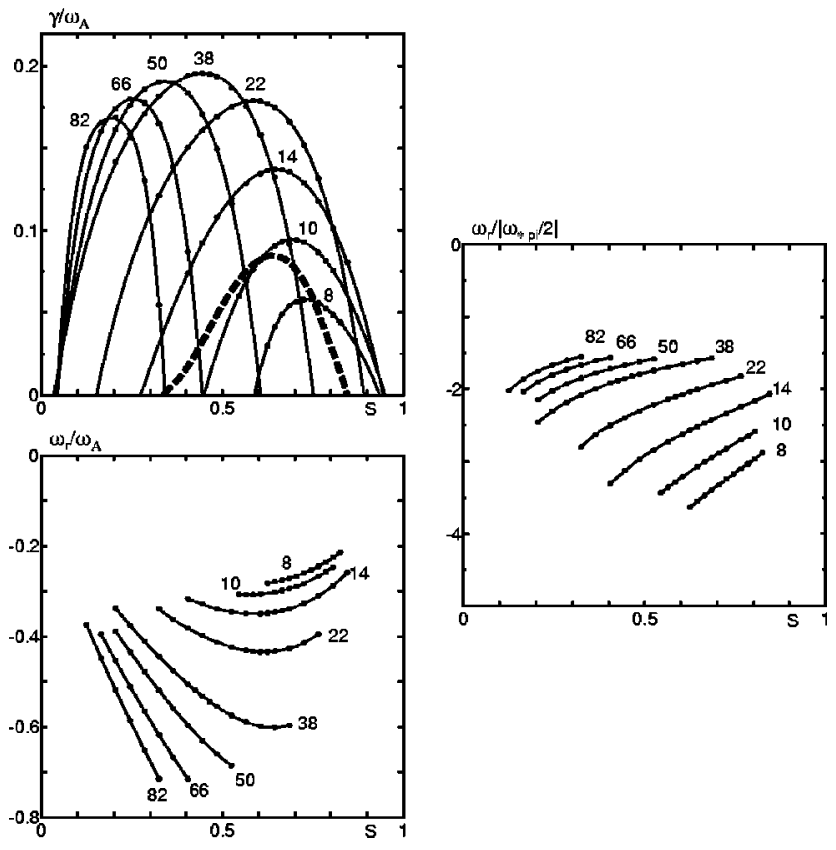

FIG. 10. Frequencies of MI modes belonging to interchange branch $\left(\theta_{k}\right.$ $=\pi$ ) as a function of $s$, in LHD with \#24 pressure at $\langle\beta\rangle \sim 1.8 \%$, for various $n_{k}$. The ideal growth rate is also shown by a dashed line. gime for our KBM equation seems to be satisfied, because $\left|\omega_{r}\right| \sim 2\left|\omega_{* p i}\right|$, so that $n_{\text {lim }}$, shown in the right frame of Fig. 4 , leads to a yield about four times the severe condition. It can be seen that the instabilities survive as $n_{k}$ becomes high, and the unstable range slides to the core region with high pressure, where the ideal modes are stable. This is a consequence of the compressibility $\propto p\left(\kappa_{d}\right)^{2}$ in the kinetic model and the curvature term plays a destabilizing role even if it is favorable, $\kappa_{d}>0$, when combined with negative $G$ at high $n_{k}$.

\section{EFFECT OF THE TRAPPED ELECTRON}

As can be seen in Eq. (3), when the fifth term is included, the equation takes the integro-differential form, i.e., the undetermined function $\xi$ is in the integration. Thus we see the effects of trapped particle perturbatively, as in Ref. 21 ,

$$
\delta \bar{\omega}=P_{t} /\left(P_{i}+P_{c}\right),
$$

with

$$
\begin{aligned}
P_{i}= & \left.\left(\frac{B_{0}}{R_{0}}\right)^{2} \frac{d I}{d \bar{\omega}}\right|_{\bar{\omega}_{0}} \int_{-\infty}^{\infty} \frac{\mathcal{A}}{B^{\theta}} \frac{\left|\xi_{0}\right|^{2}}{B^{3}} d \theta \\
P_{c}= & -\left.\frac{\mu_{0} p}{1+\tau} \frac{d G}{d \bar{\omega}}\right|_{\bar{\omega}_{0}} ^{\infty} \int_{-\infty}^{\infty} \kappa_{d}^{2} \frac{\left|\xi_{0}\right|^{2}}{B^{3}} d \theta \\
P_{t}= & \frac{\mu_{0} p \tau}{1+\tau} H\left(\bar{\omega}_{0}\right) \int_{1 / B_{\max }}^{1 / B_{\min }} d \Lambda \Lambda \\
& \times \sum\left|\int_{\theta_{1}}^{\theta_{2}} \frac{\kappa_{d} \xi_{0}}{B \sqrt{1-\Lambda B}} d \theta\right|^{2} / \int_{\theta_{1}}^{\theta_{2}} \frac{d \theta}{B \sqrt{1-\Lambda B}}
\end{aligned}
$$

Here $\bar{\omega}_{0}$ and $\xi_{0}$ is the frequency and eigenfunction of MI mode, and $\delta \bar{\omega}$ represents the variation of the frequency from $\bar{\omega}_{0}$ by including the trapped electron term. The sum is taken for the sets of turning points, and $P_{i}$ is multiplied by the factor of $\left(B_{0} / R_{0}\right)^{2}$ for our normalization in Sec. V.

In Fig. 11, the resulting growth rates perturbed by the trapped electron term are shown, together with the MI growth rates. For all $n_{k}$, the effect is found to be slightly stabilizing, as in tokamak plasmas. ${ }^{10}$ It can be seen that the ballooning-like MI modes $\left(\theta_{k}=0\right)$ are more affected by the trapped electrons than the interchange-like one $\left(\theta_{k}=\pi\right)$. This is reasonable, since the trapped particles are mostly located in the outboard of the torus. In addition, the ballooning-like modes are more localized, so that modes are apt to resonate the bounce motion of trapped particles.

At all events, it is found that the variation is very small; $\delta \bar{\gamma} / \bar{\gamma}_{0}$ is a few percent. This may be due to the fact that the eigenfunction of the KBM in LHD is extended over several helical ripples, even for the ballooning-like modes. Thus it seems to be enough to neglect the effect of the trapped electrons for the KBM stability in LHD, insofar as we use Eq. (3). It is noted that the eigenfunction of the trapped electron mode (TEM) in LHD shown in Fig. 4 of Ref. 37 seems to be much more localized than that here in Fig. 9. Of course, the change of the marginal stability cannot be seen by the per- 


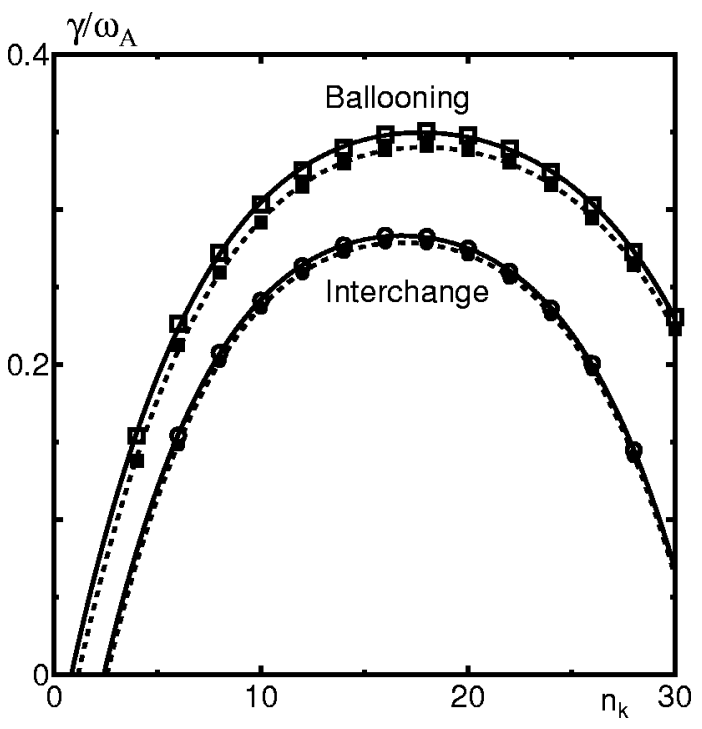

FIG. 11. MI growth rates (solid line) and its variation due to the trapped electron effect (dashed line), as a function of $n_{k}$. The ballooning and interchange-like modes are shown by squares and circles, respectively. Equilibrium and parameters are same as in Fig. 6 .

turbative method. In fact, the effects of the trapped particles may become more significant for the modes in the lower frequency regime. A more formal treatment would be required to consider such situations.

\section{COMPARISON WITH A COMPARABLE TOKAMAK}

Here the KBM results of MI modes are compared in between LHD and a circular tokamak. For the tokamak, $R_{0}$ $=3.9[\mathrm{~m}]$ and $a=0.57[\mathrm{~m}]$ is assumed, which is comparable to the LHD. The safety factor is assumed as $1 \lesssim q \lesssim 3$, from the axis to the edge, and the Mercier stability is assured. For both LHD and tokamaks, the pressure profile is \#14. Here we concentrate on the localized ballooning type mode with $\theta_{k}$ $=0$.

In Fig. 12, the ideal growth rate is plotted for a tokamak at $\langle\beta\rangle \sim 1.2 \%$ and LHD at $\langle\beta\rangle \sim 2.4 \%$, where the beta value is chosen such that the order of the growth rate is comparable. In Fig. 13, the frequencies of the KBM in both configurations at $s=0.635$ are shown as a function of $n_{k}$. The dotted line is shown for the MI-1 model and the solid line is shown for the MI model. Here $\omega_{* i} / n_{k}$ and $\eta_{i}$ are artificially taken to be the same for the tokamak and LHD in order to make the comparison easy. Since the frequency of the MI-1 modes is uniquely determined by $\gamma_{\mathrm{MHD}}$ and $\omega_{* p i} \equiv \omega_{* i}(1$ $+\eta_{i}$ ), the MI-1 curves for both configurations almost coincide.

On the other hand, the frequencies of the MI modes are not similar in both configurations. Whereas the MI mode in the tokamak is not so different from the MI- 1 mode, the MI mode in LHD is very different from the MI-1 mode. This is ascribed to the difference of the curvature, since the difference between the MI and MI- 1 models is a compressible term, which is proportional to the square of the curvature term, $\kappa_{d}^{2}$. It can be understood that the order of $\kappa_{d}$ for typical helical systems can become locally larger than that of com-

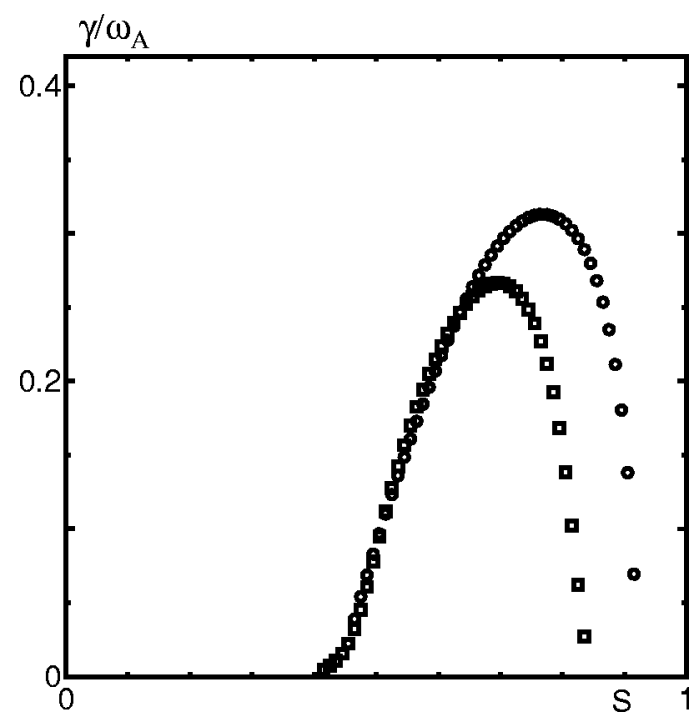

FIG. 12. Ideal ballooning $\left(\theta_{k}=0\right)$ growth rate as a function of $s$, in tokamak at $\langle\beta\rangle \sim 1.2 \%$ (circles) and LHD at $\langle\beta\rangle \sim 2.4 \%$ (squares) with \#14 pressure.

parable tokamaks, because the helical plasma is twisted and has the local ridge. It is interesting to note that similar results were found for the ion temperature gradient (ITG) modes in the two-fluid model, ${ }^{8}$ although the equations for the KBM and ITG are rather different.

As can be understood from the above discussions, the deviation of the MI frequencies from the MI-1 frequencies is dependent on the relative magnitude of the inertia to the compressible term. For the small growth rate and moderate real frequency, the inclusion of the compressible term will affect the results significantly because the magnitude of the inertia term is not so large. On the other hand, when the
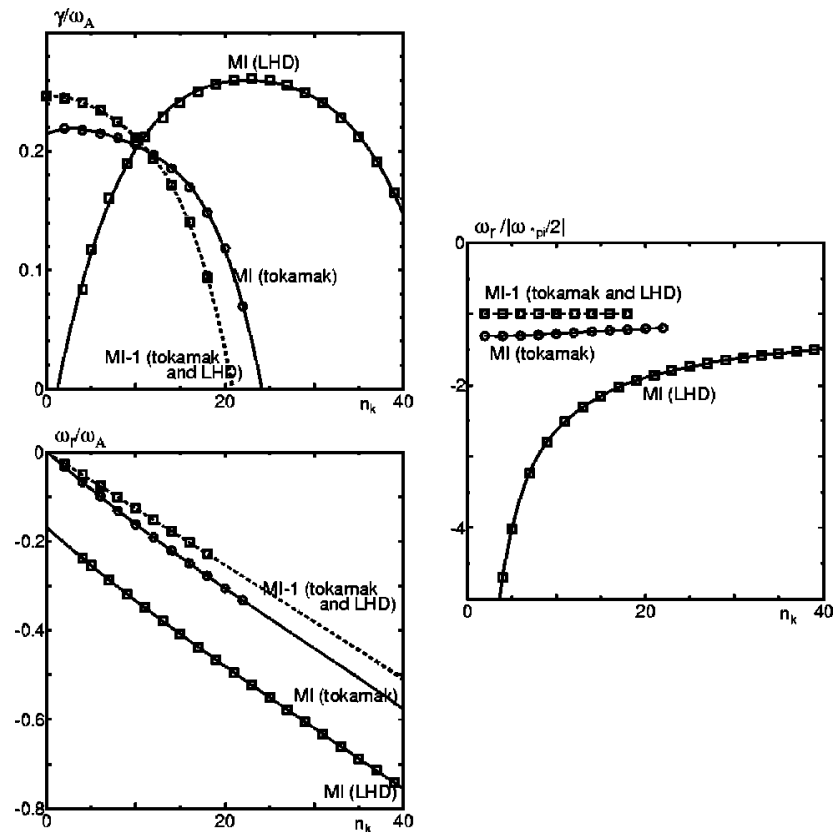

FIG. 13. Frequencies of ballooning-like modes $\left(\theta_{k}=0\right)$ as a function of $n_{k}$, in tokamak (triangles) and LHD (squares) with \#14 pressure, at $s=0.635$. The MI-1 frequencies are plotted by dashed lines and the MI frequencies are plotted by solid lines. 
order of the real frequency becomes large with $n_{k}$, the inertia term dominates the compressible term, and the dependence of the MI growth rates on $n_{k}$ would become similar to those of MI-1 growth rates as the real frequencies normalized to $\left|\omega_{* p i} / 2\right|$ tend to be unity, as can be seen in Fig. 6 or 13. Furthermore, since the $\kappa_{d}$ term is secular, the extended modes along the field line would be more affected by the compressibility. This may explain the results in Fig. 6 that the interchange-like MI modes are apt to be more unstable than the ideal modes. Physically, the finite perpendicular fluid compressibility can cause the particle density to increase in the narrow flux tubes. Since the eigenfunction in the KBM equation is closely related to the circulating electrons or perturbed parallel currents, ${ }^{10}$ this allows the mode to be driven more. In fact we can see that the ratio of the imaginary part to the real part of eigenfunction, which should be unity in the ideal case, becomes larger than unity at finite $n_{k}$, where the picture of the kinetics comes to front.

\section{CONCLUSIONS}

By solving the standard KBM equation numerically, the kinetic effects on the ideal ballooning and interchange/ Mercier modes are investigated in an $L=2$ heliotron, LHD. We show the growth rate for the finite toroidal mode number $n_{\text {tor }}$ by the quantization condition, in the case that only ion FLR effects are included (MI-1 model). As is the ideal case, the quantized growth rate becomes small at the low $n_{\text {tor }}$, in addition to the FLR stabilizing effects at the moderate $n_{\text {tor }}$. When the finite electron compressibility effects are included (MI model), the stability can change further. For the low $n_{k}$, with $n_{k}$ being the input toroidal mode number, the compressibility is found to be stabilizing as in the ideal case. Thus the low- $n$ KBM instabilities are expected to be rather weaker than the high- $n$ ideal ballooning/Mercier instabilities, due to the quantization plus compressible effects. On the other hand, it is found that the plasma core region with high pressure becomes unstable for high- $n_{k}$ modes, which is a consequence of the destabilizing compressibility. The compressibility turns into destabilizing as $n_{k}$ increases, so that the competition between the stabilizing ion FLR effects and the destabilizing electron compressibility determines the stability of higher $n_{k}$ modes in the KBM equations. From the comparison of results in between tokamaks and LHD, the compressibility influences the LHD more than the tokamak; the low- $n_{k}$ modes are more stable, while the high- $n_{k}$ modes are more unstable in LHD. This may be partly due to the larger magnitude of the local curvature of the LHD than that of a comparable tokamak. The effect of trapped electrons is found to be small, so that it seems to be reasonable to neglect it in LHD, at least for the stability of the KBM in the intermediate frequency regime.

For the low- $n$ MHD-like modes, our results are consistent with the experimental results ${ }^{24}$ in that the activity is weaker than that expected by the local/global MHD stability. ${ }^{23,29-31,38}$ However, we cannot explain how dangerous the modes with more than moderate toroidal mode number are. It has also been reported that the instabilities are relaxed in the nonlinear stage in LHD. ${ }^{39}$ It is desirable that such modes be investigated experimentally.

We use the standard KBM equation in this study. However, there are many versions of the KBM equations with many different approximations. ${ }^{9,13,15,16,33}$ For example, when the mode frequency becomes very low and the frequency is out of the intermediate range, we must use other equations valid in the low frequency regime. Moreover, for the sufficiently extended mode, such as the interchange mode near the marginal stability, the assumption $\omega_{d} / \omega \ll 1$, which is imposed in deriving the KBM equation, maybe invalid, ${ }^{33}$ where $\omega_{d}$ is the magnetic drift frequency. The comparison of the results by other equations will be our future work.

\section{ACKNOWLEDGMENTS}

The authors wish to thank Professor Greg Rewoldt, Professor Katsuji Ichiguchi and Professor Fumimichi Sano for their useful comments and suggestions.

${ }^{1}$ J. P. Freidberg, Ideal Magnetohydrodynamics (Plenum, New York, 1987). ${ }^{2}$ G. Rewoldt, W. M. Tang, and M. S. Chance, Phys. Fluids 25, 480 (1982). ${ }^{3}$ G. Rewoldt, L.-P. Ku, W. M. Tang, H. Sugama, N. Nakajima, K. Y. Watanabe, S. Murakami, H. Yamada, and W. A. Cooper, Nucl. Fusion 42, 1047 (2002).

${ }^{4}$ G. Rewoldt, L.-P. Ku, and W. M. Tang, Phys. Plasmas 6, 4705 (1999).

${ }^{5}$ T. Kuroda, H. Sugama, R. Kanno, and M. Okamoto, J. Phys. Soc. Jpn. 69, 2485 (2000).

${ }^{6}$ A. Kendle and H. Wobig, Phys. Plasmas 6, 4714 (1999).

${ }^{7}$ J. L. Lewandowski, Plasma Phys. Controlled Fusion 40, 283 (1998).

${ }^{8}$ J. Anderson, T. Rafiq, M. Nadeem, and M. Persson, Phys. Plasmas 9, 1629 (2002).

${ }^{9}$ W. M. Tang, J. W. Conner, and R. J. Hastie, Nucl. Fusion 20, 1439 (1980).

${ }^{10}$ C. Z. Cheng, Nucl. Fusion 22, 773 (1982).

${ }^{11}$ R. J. Hastie and K. W. Hesketh, Nucl. Fusion 21, 651 (1981).

${ }^{12}$ R. R. Dominguez and R. W. Moore, Nucl. Fusion 26, 85 (1986).

${ }^{13}$ A. Hirose, L. Zhang, and M. Elia, Phys. Plasmas 2, 859 (1995).

${ }^{14}$ W. M. Tang, G. Rewoldt, C. Z. Cheng, and M. S. Chance, Nucl. Fusion 25, 151 (1982).

${ }^{15}$ P. Andersson and J. Weiland, Phys. Fluids 31, 359 (1988).

${ }^{16}$ B.-G. Hong, W. Horton, and D.-I. Choi, Phys. Fluids B 1, 1589 (1989).

${ }^{17}$ H. Nordman, B. Jhowry, and J. Weiland, Phys. Fluids B 5, 3465 (1993).

${ }^{18}$ M. Yamagiwa, A. Hirose, and M. Elia, Plasma Phys. Controlled Fusion 39, 531 (1997).

${ }^{19}$ P. B. Snyder and G. W. Hammett, Phys. Plasmas 8, 3199 (2001).

${ }^{20}$ H. Qin, W. M. Tang, and G. Rewoldt, Phys. Plasmas 6, 2544 (1999).

${ }^{21}$ R. Sanchez, J. A. Jimenez, and L. Garcia, Nucl. Fusion 38, 1511 (1998).

${ }^{22}$ O. Kaneko, A. Komori, H. Yamada et al., Phys. Plasmas 9, 2020 (2002).

${ }^{23}$ K. Ichiguchi, T. Nishimura, N. Nakajima, M. Okamoto, S. Oikawa, and M. Itagaki, Nucl. Fusion 42, 557 (2002).

${ }^{24}$ S. Sakakibara, H. Yamada, K. Y. Watanabe et al., Plasma Phys. Controlled Fusion 44, A217 (2002).

${ }^{25}$ Y. Nakamura, T. Matsumoto, M. Wakatani et al., J. Comput. Phys. 128, 43 (1996).

${ }^{26}$ S. P. Hirshman, Phys. Fluids 26, 3553 (1983).

${ }^{27}$ N. Ohyabu, H. Yamada, K. Ida, K. Watanabe, H. Funaba, T. Morisaki, and LHD Experimental Group, Plasma Phys. Controlled Fusion 44, A211 (2002).

${ }^{28}$ R. L. Dewar and A. H. Glasser, Phys. Fluids 26, 3038 (1983).

${ }^{29}$ W. A. Cooper, D. B. Singleton, and R. L. Dewar, Phys. Plasmas 3, 275 (1996).

${ }^{30}$ N. Nakajima, Phys. Plasmas 3, 4556 (1996).

${ }^{31}$ P. Cuthbert, J. L. V. Lewandowski, H. J. Gardner, M. Persson, D. B. Singleton, R. L. Dewar, N. Nakajima, and W. A. Cooper, Phys. Plasmas 5, 2921 (1998).

${ }^{32}$ O. Yamagishi, Y. Nakamura, and K. Kondo, Phys. Plasmas 8, 2750 (2001).

${ }^{33}$ C. Z. Cheng, Phys. Fluids 25, 1020 (1982).

${ }^{34}$ W. D. D'haeseleer, W. N. G. Hitchon, J. D. Callen, and J. L. Shohet, Flux 
Coordinates and Magnetic Field Structure (Springer-Verlag, Berlin, 1991).

${ }^{35}$ W. H. Press, S. A. Teukolsky, W. T. Vetterling, and B. P. Flannery, $N u-$ merical Recipes in Fortran, Second Edition (Cambridge U.P., Cambridge, 1992).

${ }^{36}$ M. Kotschenreuther, Phys. Fluids 29, 2898 (1986).
${ }^{37}$ G. Rewoldt, L.-P. Ku, W. M. Tang, H. Sugama, N. Nakajima, K. Y. Watanabe, S. Murakami, H. Yamada, and W. A. Cooper, Phys. Plasmas 7, 4942 (2000).

${ }^{38}$ J. Chen, N. Nakajima, and M. Okamoto, Phys. Plasmas 6, 1562 (1999).

${ }^{39}$ H. Miura, T. Hayashi, and T. Sato, Phys. Plasmas 8, 4870 (2001). 